\title{
Procedural Knowledge, Decision Making and Performance in Women's Volleyball According to Age Group and Specific Experience
}

\author{
Rui Manuel Flores Araújo", José Afonso Neves and Isabel Maria Ribeiro Mesquita
}

Center of Research, Education, Innovation and Intervention in Sport, Faculty of Sport, University of Oporto, Portugal

\begin{abstract}
The purpose of this study was to analyze the procedural knowledge in action, decision-making and performance of the left-side attacker according to age group and specific experience, in women's volleyball. Four Portuguese women's teams participated in the present study: one under-16, one under-18 and two adult teams. A verbal interview protocol was implemented immediately after randomly chosen live game actions. The left-side attackers had to verbally respond to this question immediately following the execution of an attack, in 6 vs. 6 situations in practice. Players' decision-making and performance were analyzed by video images. Procedural knowledge was analyzed trough non-parametric statistics, namely Kruskal-Wallis and Mann-Whitney tests. Decision-making and performance were analyzed through Chi-square $\left(\mathrm{O}^{2}\right)$ testing. Results showed that adult teams and the more experienced left-side attackers generated more goal concepts and fewer condition concepts, were more often concerned with the opponent and less with teammates. Moreover, no differences in player's performance and decision-making were observed according to age group and experience as left-side attackers. Further research should consider the characterization of the training process, mainly focusing on the tactical contents, as the information provided could provide a better and deeper understanding about the players' tactical development.
\end{abstract}

Keywords: Expertise, Female Volleyball, Knowledge, Left-side attacker, Tactical indicators, Team Sports.

\section{INTRODUCTION}

Performance in sport represents a complex interaction of biological, cognitive, psychological and socio-cultural variables [1]. The study of indicators affecting performance and determining to which degree they can be changed by the training process will afford valuable insights in order to help players to optimize their potential. The open and everchanging nature of actions typical of team sports, as well as the constant need to make decisions throughout the match, influences the importance attached to the cognitive components [2]. As such, in the context of team sports, an expert player is conceived as a performer who is able to choose the appropriate action at each moment of the match, holding it in an efficient and consistent manner [3]. Therefore, knowledge and decision-making (DM] are considered important factors in the development of expertise in team sports [4].

Research comprising specific actions or game situations in different sports has been consistently showing the existence of a significant and positive correlation between players' knowledge and decision-making as well as with game performance [5]. In the literature [4], three types of knowledge are usually distinguished: declarative ("what to do"), related to factual information that can be recovered as it is in memory; procedural ("how"), reporting how to act and how to carry out the intended action that can be expressed both in execution and in the process of selecting a response;

\footnotetext{
*Address correspondence to this author at the Faculdade de Desporto da Universidade do Porto Rua Dr. Plácido Costa, 91, 4200.450, Porto, Portugal; Tel: +351225074776; Fax: +351225500689; E-mail: raraujo@fade.up.pt
}

and strategic ("why" and "when"), concerning action' rules, i.e. formulas for action. Procedural knowledge includes the appropriate response selection within a game situation [6]. Nonetheless, it is necessary to distinguish response selection and motor execution [2]. In this way, a player can have a specific knowledge about how to perform a skill (cognitive component), but still not be able to execute that skill (execution component), which can influence his decision [3].

The expert-novice paradigm has been dominant in the sport expertise research [5, 7-10]. Findings indicate that the knowledge of expert players (namely classified according to their experience) in relation to the novices is larger, deeper, better planned, more structured and organized and more sophisticated. Players with higher experience have a higher degree of declarative and procedural knowledge than players with lower experience [11]. Moreover, experts know how and when to use that knowledge, identifying, remembering and/or efficiently manipulating the relevant information at any time, allowing them a faster and more successful decision-making $[12,13]$.

Previous studies have differentiated experts from novices mainly through divergences in age, years of experience, competition experience and players' ranking in their leagues [8-10]. In spite of the analysis of the extreme groups (i.e., experts and novices) having promoted valuable information to the research and practical domain this approach presents some limitations. Indeed, the analysis of the tactical indicators must be carried throughout the player's development as a continuous process, in order to define a pathway that could 
afford useful information for training and competition. Hence, more research is needed to examine procedural knowledge and decision-making between closer groups in order to differentiate possible changes throughout this process and thereby to give useful information for training process [14].

In the case of volleyball, research follows the same tendencies of research applied in other sports [17-21]. Although expertise is sport-specific $[8,9]$ and, within it, function- and task-specific $[15,16]$ research has been disregarding this important requirement. As such, it becomes important to consider experience in the specific function within the game in procedural knowledge, decision-making and game performance analysis. The attack action demands players to skilfully choose the best solution in each game situation [22]. Currently, the attack is referred as the most determinant game action for the teams' performance [23]. Within the attack action, the left-side attacker is considered the most requested player at the highest level [24]. This requires a powerful left-side attacker and also a sound tactical reasoning, upon which the quality of his decision-making will depend. Therefore, the purpose of this study was to compare the procedural knowledge in action, player's performance and decision-making of left-side attackers according to age group and specific experience, in women's volleyball.

\section{METHODS}

\section{Participants}

The sample comprised four women's teams from Portugal: one under-16 $(\mathrm{n}=6)$, one under-18 $(\mathrm{n}=6)$ and two adult teams $(n=8)$. Overall, 20 female left-side players were interviewed in a total of 123 reports. All the interviewed players were fully informed about the purposes of the study, why it was being undertaken, and how it would be disseminated and used. Additionally, participants retained the right to withdraw at any time. All of players that participated in the present study had at least 4 practices of two hours per week. Additionally, the two adult teams under study were competing in the $1^{\text {st }}$ division of the Portuguese Volleyball Federation, whilst the other two teams were competing in the under-16 and under-18 Portuguese National Championships.

\section{Variables and Data Collect}

Two independent variables were considered: specific experience and age group. Regarding specific experience, players were divided according to the number of years of practice as left-side attackers. Ten years is frequently used to differentiate novices and experts. However, in the present study 5 years was the criterion used in order to represent closer groups of player's experience, and two groups were considered:less experienced players (players with less than 5 years of practice; $\mathrm{n}=13,65 \%$ ) and more experienced players (with more than 5 years of practice as left-side attackers; $\mathrm{n}=7,35 \%$ ). Relatively to age group, players were divided into three groups: under-16 $(\mathrm{n}=6,30 \%)$, under-18 $(\mathrm{n}=6$, $30 \%)$, and adult teams $(\mathrm{n}=8,40 \%)$, according to the practice levels defined by the Portuguese Volleyball Federation.

On other hand, three dependent variables were considered: procedural knowledge, decision-making and performance. A verbal interview protocol adapted from McPherson
[10] was implemented for the evaluation of the procedural knowledge in action of the left-side attackers during the competition. This protocol was conducted immediately after randomly chosen live game actions, more specifically after attack actions as recommend by McPherson [10], in a minimum of 4 and maximum of 6 attacks for each player. This type of protocol was also implemented in research within different sports, such as: tennis [8-10, 25, 26], base ball [27], and volley ball [17]. These aforementioned studies applied a verbal interview to be implemented in simulated game situations in order to identity "which" information the players attended to and "how" they used it. The interview consisted in one broad question - "What were you thinking about while playing that point?" The left-side attackers had to verbally respond to this question immediately following the execution of an attack, in 6 vs. 6 situations in practice. The game was not interrupted, since a substitute player immediately entered the field, and stayed there while the other player was being interviewed. The attacker's responses were recorded on audiotape using an .mp3 recorder, which were later transcribed and coded.

The verbal reports were literally transcribed and a content analysis was carried out using a protocol model originally structured for tennis $[8,9]$ and later adapted to the requirements of volleyball [17]. The units of information were classified according to five main conceptual categories: goal concepts, condition concepts, action concepts, regulatory concepts, and do concepts [8]. Due to the reduced number of both regulatory and do concepts, and taking into account their similarity to action concepts, in the present study they were grouped into one functional unit, named action concepts. Therefore, in the present study three conceptual categories were considered:

a) Goal concepts: reflecting the means by which the game was won, the purpose of a chosen action, or the specification of a condition referring to the game's goal structure (e.g., "getting the ball in", "exploit the block");

b) Condition concepts: specifying when or under what conditions to apply the action or patterns of action to achieve the goal (e.g., the opponent position on court, opponent's weakness);

c) Action concepts: referring to the selected action or patterns of action that may produce goal-related changes in the context of a sport situation (e.g., "move to the net"). These concepts also specify whether the intended action was carried out or not (e.g., a statement that indicates the participant failed to execute a serve), and how to perform the action (statement that includes mechanical labels about how to execute the action).

Goal, condition and action concepts were also examined for concept sophistication. In the research by McPherson[8$10]$ in tennis, three-goal hierarchy levels were differentiated. In this study, hierarchical levels of goals and conditions subconcepts about own team members were also analyzed as they are relevant in team sports [17]: (i) level 0: player's sub concept goals about themselves; (ii) level 1: player's sub concept goals about team members; (iii) level 2: player's sub concept goals about their opponent; (iv) level 3: sub concept goals of other nature (reduced ' $n$ '). Concerning the evalua- 
tion of the players' performance, 479 attack actions were analyzed. The attack's efficacy was measured trough [28]: Kill attack (KILL) - conquering the point; Error (ERR) direct error or blocked attack; Neutral attack (NEU) - attack easily defended by the opponent or recovered by the own team after block coverage; ATP - attack posing difficulties on the opponent's defence, although not automatically resulting in a point. From these attacks, 123 were withdrawn to decision-making analysis. Three independent observers (expert volleyball coaches) evaluated the players' decisionmaking from 123 game actions. According to the setting action and the opponent block these expert coaches classified each action as appropriate or inappropriate, even if this action resulted in an error. The action was deemed appropriate if two out of three expert coaches classified it in that way. The expert volleyball coaches reached $83 \%$ of agreements, which is in agreement with the literature [29]. These actions for decision-making evaluation corresponded to the verbal interviews. Therefore, just some parts of player's attacks were used to decision-making evaluation. Even when interviews were not realized in game situation, attack efficacy for player's performance was observed. Both decision-making and performance were analyzed by video images, usingan Asus F3JC-APO2H laptop with the Playing Media Files Software from Microsoft - Windows Media Player (version 11).

\section{Reliability}

To ensure the reliability of the codification, intraobserver (the two observations occurred with an interval of more than 15 days) and inter-observer (observation for an observer with experience in this role and knowledgeable in volleyball) codings were used. Intra-observer reliability made use of 14 interviews (11\%), more than the minimum acceptable value of $10 \%$ stipulated by the literature [30]. To measure the degree of reliability of the observations, the formula of Bellack et al. [31] was used.

Results for intra-observer reliability showed percentages of agreement above the minimum indicated, namely $80 \%$ [30]. The minimum value found was $85.7 \%$ for player's performance and action concepts and the maximum value of $100 \%$ in the variables goal concepts, goal hierarchical levels, condition hierarchical level (0) and condition hierarchical level (1). Relatively to inter-observer reliability, results also showed percentage of agreements above the threshold value of $80 \%$. The minimum value found was $87.5 \%$ for player's performance, condition concepts, action concepts and condition hierarchical level (2). The maximum value of $100 \%$ was found in goal concepts, goal hierarchical level (1), goal hierarchical level (3) and condition hierarchical level (3).

\section{Data Analysis}

Descriptive statistics and inferential statistics were applied to collected data. Procedural knowledge according to age group was analyzed through content analysis. Exploratory data analysis revealed non-normality in the distribution of the data. Therefore, registration units from this analysis were compared trough nonparametric statistics, namely the KruskalWallis test. This test was applied to compare the means of the different variables. Procedural knowledge according to specific experience was also analyzed trough content analysis, but in this case the Mann-Whitney test was applied.
Moreover, performance and decision-making were analyzed trough Chi-square $\left(\mathrm{O}^{2}\right)$ testing, with analysis of the adjusted residuals, to test the association between these variables, age group and specific experience. Statistical significance was set at $5 \%$ and all analyzes were performed in SPSS 18.0 (SPSS Inc, USA).

\section{RESULTS}

Adult teams showed higher values in goals and lower values of condition concepts than under-16 and under- 18 teams. Table 1 presents the statistically significant results according to age group. In regard to action concepts, no significant differences were found $\left(\mathrm{H}_{(2)}=0.907, \mathrm{p}=0.635\right)$. Analysing the goal hierarchical levels, statistically significant differences were observed in the hierarchical level (0) and (2) between groups.

Adult teams presented higher values of goal concepts related to themselves and goal hierarchical level (2) (related to the opponent) when compared to the under-18 teams. However, this group (adult teams) exhibited lower values of condition hierarchical level (1), than under-16 and under-18 teams. Under-16 players presented higher values than adult and under-18 teams in the condition hierarchical level (2). Moreover, adult teams presented lower values of condition quality level. No significant differences were found according to action quality level $\left(\mathrm{H}_{(2)}=3.236, \mathrm{p}=0.198\right)$.

Also, no statistically significant differences were found between the three age group groups (Table 2), according to player's performance $\left(\mathrm{O}^{2}=11.99, \mathrm{p}=0.062\right)$ and decisionmaking $\left(\mathrm{O}^{2}=4.331, \mathrm{p}=0.115\right)$. Table 3 presents the results according to players' specific experience as left-side attackers. The more experienced players showed superior values of goal concepts and fewer number of condition concepts.

Regarding action concepts, no significant differences were found $(\mathrm{F}=1656.000, \mathrm{p}=0.908)$. More experienced players also generated higher number of goal hierarchical level (0) and goal hierarchical level (2). However, these players presented fewer references of condition hierarchical level (1), condition hierarchical level (2) and condition quality level. There were no statistically significant differences between groups in player's performance $\left(\mathrm{O}^{2}=3.585, \mathrm{p}=0.31\right)$ and decision-making accuracy $\left(\mathrm{O}^{2}=3.131, \mathrm{p}=0.77\right)$ according to their specific experience as left-side attackers (Table 4).

\section{DISCUSSION}

As aforementioned, the purpose of the present study was to compare procedural knowledge in action, player's performance and decision-making of left-side attackers according to age group and specific experience in women's volleyball. Findings from the present study showed similar results according to age group and player's specific experience, which was somewhat expected since usually the most experienced players play in adult teams. Indeed, adult teams and more experienced players presented more goal concepts, in agreement with previous studies $[10,18]$, in which more experienced players also presented more goal concepts. However, in tennis experts and novices have been shown to generate a similar number of goal concepts $[8,9]$. Despite 
Table 1. Procedural Knowledge According to Age Group

\begin{tabular}{|c|c|c|c|c|c|}
\hline & & \multicolumn{2}{|c|}{ Kurskal-Wallis Test } & \multicolumn{2}{|c|}{ MEAN } \\
\hline & & $\mathbf{H}$ & Sig. & Group & $\mathbf{M}$ \\
\hline \multirow{15}{*}{ Procedural Knowledge } & \multirow{3}{*}{ Condition concepts } & \multirow{2}{*}{27.937} & \multirow{2}{*}{0.000} & 1 & 52.11 \\
\hline & & & & 3 & 78.99 \\
\hline & & 47.683 & 0.000 & 1 & 84.88 \\
\hline & \multirow{3}{*}{ Goal hierarchical level (0) } & \multirow{3}{*}{13.478} & \multirow{3}{*}{0.001} & 1 & 54.78 \\
\hline & & & & 2 & 54.78 \\
\hline & & & & 3 & 72.20 \\
\hline & \multirow[b]{2}{*}{ Goal hierarchical level (2) } & \multirow[b]{2}{*}{7.840} & \multirow[b]{2}{*}{0.020} & 1 & 60.13 \\
\hline & & & & 2 & 55.33 \\
\hline & Condition hierarchical level (1) & 34.676 & 0.000 & 3 & 42.05 \\
\hline & \multirow{6}{*}{ Condition hierarchical level (2) } & \multirow{6}{*}{20.517} & \multirow{3}{*}{0.000} & 1 & 80.79 \\
\hline & & & & 2 & 64.17 \\
\hline & & & & 3 & 47.21 \\
\hline & & & \multirow{3}{*}{0.000} & 1 & 70.24 \\
\hline & & & & 2 & 67.17 \\
\hline & & & & 3 & 40.08 \\
\hline
\end{tabular}

Legend: Group 1 - under-16 teams; Group 2 - under-18 teams; Group 3 - adult teams

\section{Table 2. Decision-Making and Players' Performance According to Age Group}

\begin{tabular}{|c|c|c|c|c|c|c|c|c|c|}
\hline & \multicolumn{3}{|c|}{ Decision-making } & \multicolumn{5}{|c|}{ Players' Performance } \\
\hline & & $\mathbf{A}$ & $\mathbf{I}$ & Total & ATP & ERR & KILL & NEU & Total \\
\hline \multirow{2}{*}{$\mathrm{U}-16$} & $C(E)$ & $23(24)$ & $13(12)$ & 36 & $10(7.3)$ & $8(7.3)$ & $5(12.6)$ & $13(8.8)$ & 36 \\
\hline & $\mathrm{AR}$ & -0.4 & 0.4 & & 1.3 & 0.3 & -3.2 & 1.9 & \\
\hline \multirow{2}{*}{$\mathrm{U}-18$} & $C(E)$ & $20(24)$ & $16(12)$ & 36 & $7(7.3)$ & $8(7.3)$ & $16(12.6)$ & $5(8.8)$ & 36 \\
\hline & $\mathrm{AR}$ & -1.7 & 1.7 & & -0.2 & 0.3 & 1.4 & -1.7 & \\
\hline \multirow{2}{*}{ Adult } & $C(E)$ & $39(34)$ & $12(17)$ & 51 & $8(10.4)$ & $9(10.4)$ & $22(17.8)$ & $12(12.4)$ & 51 \\
\hline & AR & 1.9 & -1.9 & & -1.1 & -0.6 & 1.6 & -0.2 & \\
\hline \multicolumn{2}{|c|}{ Total } & 82 & 41 & 123 & 25 & 25 & 43 & 30 & 123 \\
\hline
\end{tabular}

Legend: A - appropriate decisions; I - inappropriate decisions.

\section{Table 3. Procedural Knowledge According to Specific Experience}

\begin{tabular}{|c|c|c|c|c|c|}
\hline & & \multicolumn{2}{|c|}{ Mann-Whitney Test } & \multicolumn{2}{|c|}{ MEAN } \\
\hline & & $\mathbf{U}$ & Sig. & Group & $\mathbf{M}$ \\
\hline \multirow{5}{*}{ 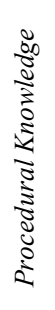 } & \multirow{2}{*}{ Goal concepts } & \multirow{2}{*}{1035.000} & \multirow{2}{*}{0.000} & 1 & 54.47 \\
\hline & & & & 2 & 77.63 \\
\hline & \multirow{2}{*}{ Condition Concepts } & \multirow{2}{*}{773.500} & \multirow{2}{*}{0.000} & 1 & 72.68 \\
\hline & & & & 2 & 39.84 \\
\hline & Goal hierarchical level ( 0 ) & 1291.000 & 0.006 & 2 & 71.22 \\
\hline
\end{tabular}


Table 3. contd...

\begin{tabular}{|c|c|c|c|c|}
\hline & \multicolumn{2}{|c|}{ Mann-Whitney Test } & \multicolumn{2}{|c|}{ MEAN } \\
\hline \multirow{2}{*}{ Goal hierarchical level (2) } & \multirow{2}{*}{1382.500} & \multirow{2}{*}{0.012} & 1 & 58.66 \\
\hline & & & 2 & 68.94 \\
\hline \multirow{2}{*}{ Condition hierarchical level (1) } & \multirow{2}{*}{916.000} & \multirow{2}{*}{0.000} & 1 & 70.96 \\
\hline & & & 2 & 43.40 \\
\hline Condition hierarchical level (2) & 12.02 & 0.010 & 2 & 50.55 \\
\hline \multirow{2}{*}{ Condition quality level } & \multirow{2}{*}{808.000} & \multirow{2}{*}{0.000} & 1 & 65.40 \\
\hline & & & 2 & 41.09 \\
\hline
\end{tabular}

Legend: Group 1 - less experienced players ([0-5 years of practice as left-side attackers); Group 2 - more experienced players (more than 5 years of practice as left-side attackers).

Table 4. Decision-Making and Players' Performance According to Specific Experience

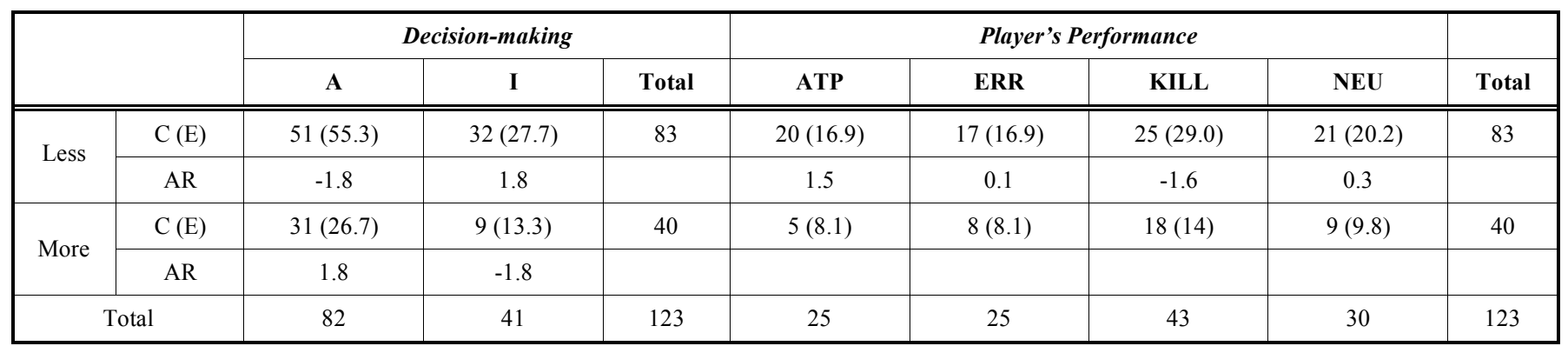

Legend: A - appropriate decisions; I - inappropriate decisions; Less - less experienced players; More - more experienced players

both tennis and volleyball being net sports, the open and changing nature of teams sports [2] provides more indicators to make decisions, which may explain the different results of volleyball and tennis studies. In the same vein, goals presented by the adult teams and more experienced players were related to the opponent, supporting previous research with left-side attackers [18] and setters [21]. Mesquita \& Graça [21] studied an elite volleyball setter and recognized the exploration of opponent's weakness as characteristics of an exceptional setter.

Our data showed that the more experienced players and the adult teams referred fewer conditions and more often goals, corroborating the findings of Araújo, Afonso, \& Mesquita [18] with left-side attackers, which also found more often goals and less often conditions. However, Moreno et al. [19] stated that expert setters generated more total conditions than novices. These differences hint that there is an influence of specific function within the game. While setting requires high analysis of conditions [21], the attack action is more oriented to the finalization, and consequently to the goals. In addition, it is possible that, in relation to attackers, conditions concepts were implied within the goals, as the conditions, even though not being mentioned, could be implied in these reports (e.g., when the player's goal is to exploit the opponent block, this athlete has to know how it is formed, despite not referring this condition). On the other hand, less experienced players could refer more conditions without them being necessarily relevant or appropriate [20]. Hence, these results suggest that concepts' quantity is not synonymous of quality, since experts are more selective and know how and when to use that knowledge, identifying, remembering and/or efficiently manipulating the relevant information at any time $[5,13]$.

Concerning player's performance and decision-making no differences were found in this study, in disagreement with previous research [31]. This divergence might be explained due to group's characteristics. Indeed, Vaeyens et al. [32] studied differences in responses accuracy between soccer players and non-players [very distinctive groups) and found that the first ones made more accurate decisions. Therefore, the game complexity differs according to the age group and specific experience as left-side attacker, thus requiring the appropriation of decision-making and performance to their play level. In fact, increases in body size and accumulation of practice lead to better performance of sport skills [14]. In addition, at older ages more complex skills are added, and the rules for competition become more complex [14]. As such, much more research considering other factors, namely game level, is needed to examine possible transitions in sport skill and game complexity during later childhood and adolescence. Moreover, the results obtained in relation to decision-making can find explanation on the instrument employed that could not be sensitive for appreciate differences in decision-making in a high level game. Results obtained regarding players' performance could also find explanation in the quality of players (attacker, opponent block, defenders, etc.) and previous actions to attack, namely setting and serve-reception actions. 
Overall, the findings of the present study were somewhat contradictory to previous research. Explanations for such discrepancies might be found on different factors, such as the specific function within the game interfering with the indicators that players consider important to situational analysis. For instance, goals are more important to the attack and conditions are more important to the setting action. Therefore, this factor should be considered in future studies in order to avoid abusive extrapolations. Moreover, studies have compared opposite groups (experts and novices). However, the present study analyzed closer groups, which could allow a better understanding of these issues in the player's long-term development. Therefore, longitudinal studies are suggested, affording the analysis of the tactical evolution of the players taking into account the training process to which they are subject. Therefore, these considerations suggest the necessity of studies that combine qualitative and quantitative analysis or qualitative research based on case studies, since fruitful information could be reached from the actual environment and conditions where players learn and apply tactical contents.

\section{CONCLUSIONS}

Findings from the present study showed similar results in procedural knowledge, decision-making and player's performance according to age group and player's specific experience. The results suggested that adult teams and more experienced players play more under goal than condition. In addition, goals presented for adult teams and more experienced players were more oriented towards the opponent. The present study also showed no differences in player's performance and decision-making according to age group and experience as left-side attackers.From this study, relevant implications can be withdrawn for the process of training since players should have opportunity to skill development and to practice decisions. However, future studies should contemplate other variables, such as the training orientation and the participants' game level. Therefore, studies that combine qualitative and quantitative analysis should be realized, as well as longitudinal studies that could analyze player's tactical evolution.

\section{CONFLICT OF INTEREST}

The authors confirm that this article content has no conflicts of interest.

\section{ACKNOWLEDGEMENT}

Declared none.

\section{REFERENCES}

[1] Tenenbaum G. Expert athletes. An integrated approach to decision making. In: Starkes J, Ericsson KA, Eds. Expert performance in sports advances in research on sport expertise. Champaign (Illinois): Human Kinetics 2003; pp. 192-218.

[2] Thomas K. The development of sport expertise: from Leeds to MVP legend. Quest 1994; 46(2): 199-210.

[3] Gréhaigne J-F, Godbout P, Bouthier D. The teaching and learning of decision making in team sports. Quest 2001; 53: 59-76.

[4] Thomas K, Thomas J. Developing expertise in sport: the relation of knowledge and performance. Int J Sport Psychol 1994; 25: 295312 .
[5] Iglesias D, Moreno MP, Santos-Rosa FJ, Cervelló E, Del Villar F. Cognitive expertise in sport: relationships between procedural knowledge, experience and performance in youth basketball. J Hum Mov Stud 2005; 49: 65-76.

[6] McPherson S, French K. Changes in cognitive strategies and motor skill in tennis. J Sport Exerc Psychol 1991; 13(1): 26-41.

[7] Del Villar F, García L, Iglesias D, Moreno MP, Cervelló E. Expertnovice differences in cognitive and execution skills during tennis competition. Percept Mot Skills 2007; 104: 355-65.

[8] McPherson S. Tactical differences in problem representations and solutions in collegiate varsity and beginner female tennis players. Res Q Exerc Sport 1999;70(4):369-84.

[9] McPherson S. Expert-novice differences in performance skills and problem representations of youth and adults during tennis competition. Res Q Exerc Sport 1999; 70(3): 233-51.

[10] McPherson S. Expert-novice differences in planning strategies during collegiate singles tennis competition. J Sport Exerc Psychol 2000; 22(1): 39-62.

[11] Thomas J, French K, Thomas T, Gallagher J. Children's knowledge development and sport performance. In: Smoll FL, Magill RA, Ash MJ, Eds. Children in sport. Champaign IL: Human Kinetics 1988.

[12] Singer R, Janelle C. Determining sport expertise: From genes to supremes. Int J Sport Psychol 1999; 30: 117-50.

[13] Dodds P, Griffin L, Placek J. A selected review of the literature on development of learners' domain-specific knowledge. J Teach Phys Educ 2001; 20(4): 301-13.

[14] French K, Nevett M, Spurgeon J, Graham K, Rink J, McPherson S. Knowledge representation and problem solution in expert and novice youth baseball players. Res Q Exerc Sport 1996; 67(4): 38695.

[15] Laurent E, Ward P, Williams AM, Ripoll H. Expertise in basketball modifies perceptual discrimination abilities, underlying cognitive processes, and visual behaviours. Vis Cogn 2006; 13(2): 247-71.

[16] Smeeton N, Ward P, Williams AM. Do pattern recognition skills transfer across sports? A preliminary analysis. J Sports Sci 2004; 2(2): 205-13.

[17] Moreno MP, Moreno A, Ureña A, Iglesias D, Del Villar F. Application of mentoring through reflection in female setters of the Spanish National Volleyball team: a case study. Int J of Sport Psychol 2008; 39(1): 59-76.

[18] Araújo R, Afonso A, Mesquita I. Procedural knowledge, decisionmaking and game performance analysis in Female Volleyball's attack according to the player's experience and competitive success. Int J Perform Anal Sport 2011; 11: 1-13.

[19] Moreno MP, Moreno A, Ureña A, García L, Del Villar F. Representación de problemas tácticos en colocadoras de voleibol de las selecciones nacionales españolas: efecto de la pericia. Rev Iberoam Psicol Ejercicio Deporte 2008; 3(2): 229-40.

[20] McPherson S, Dovenmuehler A, Murray M. Player differences in representation of strategic knowledge and use during a modified volleyball blocking game situation. In: Meeting of the North American Society for the Psychology of Sport and Physical Activity, Pittsburgh, PA 1992.

[21] Mesquita I, Graça A. Probing the strategic knowledge of an elite volleyball setter: a case study. Int J Volleyball Res 2002; 5(1): 137.

[22] Papadimitriou K, Pashali E, Sermaki I, Mellas S, Papas M. The effect of the opponents' serve on the offensive actions of Greek setters in volleyball games. Int J Perform Anal Sport 2004; 4(1): 23-33.

[23] Marcelino R, Mesquita I, Afonso J. The weight of terminal actions in volleyball. Contributions of the spike, serve and block for the teams' rankings in the World League 2005. Int J Perform Anal Sport 2008; 8(2): 1-7.

[24] Araújo R, Castro J, Marcelino R, Mesquita I. Relationship between the opponent block and the hitter in elite male volleyball. J Quant Anal Sports 2010; 6(4): 1-10.

[25] Nielsen T, McPherson S. Response selection and execution skill of professional and novices during singles tennis competition. Percept Mot Skills 2001; 93: 541-55.

[26] McPherson S, Kernodle M. Mapping two new points on the tennis expertise continuum: tactical skills of adult advanced beginners and entry-level professionals during competition. J Sports Sci 2007; 25(8): 945-59. 
[27] McPherson S. The influence of player experience on problem solving during batting preparation in baseball. J Sport Exerc Psychol 1993; 15(3): 304-25.

[28] Afonso J, Mesquita I. Determinants of block cohesiveness and attack efficacy in high-level women's volleyball. Eur J Sport Sci 2011; 11(1): 69-75.

[29] Van der Mars H. Observer reliability: issues and procedures. In: Darst P, Zakrajsek D, Mancini V, Eds. Analyzing physical education and sports instrution. $2^{\text {nd }}$ ed. Champaing, IL: Human Kinetics 1989; pp. 53-79.
[30] Tabachnick BG, Fidell LS. Using multivariate statistics. $5^{\text {th }}$ ed. Boston: 18 Allyn and Bacon 2007.

[31] Bellack AA, Kliebard HM, Hyman RT, Smith FL. The Language of the Classroom. New York: Columbia University Press 1966.

[32] Vaeyens R, Lenoir M, Williams AM, Mazyn L, Philippaerts R. The effects of task constraints on visual search behavior and decisionmaking skill in youth soccer players. J Sport Exerc Psychol 2007; 29: 147-69.

(C) Araújo et al.; Licensee Bentham Open.

This is an open access article licensed under the terms of the Creative Commons Attribution Non-Commercial License (http://creativecommons.org/licenses/ by-nc/3.0/) which permits unrestricted, non-commercial use, distribution and reproduction in any medium, provided the work is properly cited. 